were analysed in a sample of 242 elderly. The disability incidence was $91.5 / 1000$ person-years in robust, $118.6 / 1000$ person-years in pre-frail, and 223.2/1000 person-years in frail elderly. Frailty is associated to the risk of developing disability $(\mathrm{OR}=3.32 ; \mathrm{p}=0.015)$. In model 1 , adjusted for age, sex and education, frailty remains significantly associated $(O R=2.54 ; p=0.045)$. In model 2 , after adding depression, falls, BMI, and mobility limitation, frailty lost significance, but still shows risk $(\mathrm{OR}=2.61 ; \mathrm{p}=0.066)$.

Conclusions Incidence rate of ADL limitation was greater in frail elderly, but other factors, as depression and falls, are also important and should be considered.

\section{P1-123 EDUCATION, MUNICIPAL DEVELOPMENT AND RATE OF HOMICIDES IN MINAS GERAIS, BRAZIL}

doi:10.1136/jech.2011.142976d.16

I C Gomes, ${ }^{*}$ E L Machado, M A Costa, G S Atuncar. Federal University of Minas Gerais, Belo Horizonte, Minas Gerais, Brazil

Introduction Homicide rates are increasingly and have become a public health problem in developing countries, affecting mainly young males aged 15-29 years. We aimed to investigate the relationship between the development level of the cities and schooling, with the rate of homicides.

Methods An ecological study was performed with data from the State of Minas Gerais, Brazil (population >50 000) in 2006. We used the following variables: homicide mortality rate per 100000 in 15-29 year olds, school attendance of young people aged 15-17 years and the Index for Social Responsibility (ISR). The ISR is a compound indicator that aggregates information from health outcomes, income, education, demography, public safety, management, housing and environment, culture and sport and leisure. The relationship between the variables was investigated by linear regression.

Results Of the 2320 deaths in Minas Gerais in 2006 due to external causes, $283(12 \%)$ were due to homicides. The victims were mostly male ( $89 \%)$ and $43 \%$ belonged to age group 15-29 years. Regression modelling showed the homicide rate decreased with increasing ISR and increasing school attendance.

Conclusion These results suggests that mortality in young people is related to lower educational level. Thus, strategies for dealing with violence should consider investing in the education of citizens.

\section{P1-124 KNOWLEDGE AND ATTITUDES TOWARDS THE HUMAN PAPILLOMAVIRUS VACCINE AMONG COLLEGE STUDENTS}

doi:10.1136/jech.2011.142976d.17

${ }^{1} \mathrm{O}$ Simwale, ${ }^{2} \mathrm{~N}$ Daneshvar, ${ }^{*} \mathrm{~L}$ Scott, ${ }^{2} \mathrm{~T}$ Sylk, ${ }^{2} \mathrm{M}$ Penn. ${ }^{1}$ Pennsylvania Department of Public Health, Harrisburg, Pennsylvania, USA; ${ }^{2}$ Franklin and Marshall College, Lancaster, Pennsy/vania, USA

Introduction Human papillomavirus (HPV) is the most common sexually transmitted infection (STI) and is a leading cause of cervical cancer in the USA; most cases occur in individuals between the ages of 15 and 24, yet few studies have examined factors associated with HPV vaccine acceptance among this age group.

Methods Responses to a 15-question web-based survey were used to determine predictors and deterrents of HPV vaccine acceptance among college students.

Results Multinomial logistic regression was used to analyse data at a CI of $95 \%$. Students who believed they were at risk for contracting HPV were over four times more likely to be willing to receive the vaccine than students who did not believe they were at risk [OR: 4.2; CI 2.113 to 8.359; $\mathrm{p}=0.000]$. Students who had previously been diagnosed with an STI were almost seven times more likely perceive they were at risk for contracting HPV [OR: 6.86; CI 1.85 to 25.52; $p=0.009]$. Male students were less willing to receive the preventative HPV immunisation than their female counterparts [OR: 0.355; CI 0.155 to $0.812 ; p=0.007]$. Students who were aware of the relationship between HPV and cervical cancer were nearly two times more likely to report willingness to receive the vaccine [OR: 1.93; CI 0.987 to $3.754 ; \mathrm{p}=0.044]$.

Conclusion HPV vaccination uptake may be increased if future programs emphasise students' susceptibility to HPV infection. Vaccination campaigns should also include more information regarding the relationship between HPV and cervical cancer, as well as vaccine safety and effectiveness.

\section{P1-125 SOCIAL INEQUALITIES IN BIOMARKERS: FINDINGS FROM THE ENGLISH LONGITUDINAL STUDY OF AGEING (ELSA)}

doi:10.1136/jech.2011.142976d.18

C de Oliveira. * University College London, London, UK

Introduction Although social inequalities in health are widely recognised, the process by which the social environment becomes translated into physiological and psychological processes that influence health remains unclear. The aim of this longitudinal study was to compare changes over time and the relationship between socioeconomic position and different biomarkers in a nationally representative sample of older adults in England.

Methods The sample consisted of 6260 participants aged 50 and older who took part in the 2004 and 2008 waves of the English Longitudinal Study of Ageing (ELSA), a prospective national cohort study of people aged 50 years and over. The analyses included biomarkers associated with cardiovascular disease as well as those associated with improved health and well-being. The changes in these markers over time were modelled using two measures of socioeconomic status: total (non-pension) wealth and level of education. Analyses were adjusted for confounding variables.

Results The prevalence of overweight, general and abdominal obesity was inversely related to socioeconomic position as defined by wealth and education. Fewer participants who were better off and had more educational qualifications had levels of 'good' cholesterol (HDL), triglycerides, fibrinogen, CRP and glycated haemoglobin that would indicate increased risk. Total and LDL cholesterol showed an inverse socio-economic gradient.

Conclusion Overall, there was a very clear socio-economic gradient in biomarkers measured in ELSA and the pattern was similar for both men and women.

\section{P1-126 NUTRITION NEEDS TO DEVELOP EFFECTIVE MEASUREMENT POLICIES}

doi:10.1136/jech.2011.142976d.19

E Alexander, ${ }^{*}$ D Yach, G A Mensah. PepsiCo, Purchase, New York, USA

Measurements of food and nutrition availability and intake inform national policy, standards and research. However, existing nutrition data are not complete and often subject to error. Accurate and comprehensive data are needed on the source of food and nutrients. Nutrient sources amenable to intervention and the cost of such interventions must be described to determine feasible approaches. Food sales data describes the formal sector, omitting the informal sector which accounts for a majority of energy intake in some countries. Food balance sheets used by the FAO to estimate dietary intake do not take into account household or retail waste, overestimating dietary intake. NHANES, the US population intake survey is subject to underestimation of dietary intake due to reporting error, especially by overweight subjects. Standard portion 